\title{
Schizophrenia in a worldwide perspective: explaining similarities and differences
}
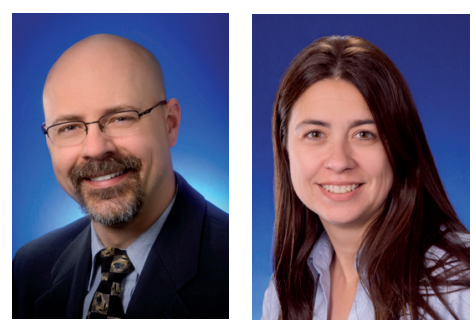

"Pharmacotherapy is the cornerstone of schizophrenia treatment. However,

not all patients have the same access to all treatments."

Jamie Karagianis ${ }^{\dagger} \&$ Angela Hill'

'Eli Lilly \& Company, Lilly Corporate Centre, Indianapolis, IN, 46285, USA

†Author for correspondence: Senior Medical Advisor, Neuroscience EMBU, Eli Lilly Canada Ino

3650 Danforth Ave, Toronto, ON, M1N 2E8, Canada = Tel.: +1 4166933878 = Fax: +1 4166997352

- karagianis_jamie@lilly.com

Schizophrenia is estimated to affect as many as one in every 250 people at some point in their lives [1]. It is the fifth leading cause of years lost through disability in men and the sixth leading cause in women [101].

Pharmacotherapy is the cornerstone of schizophrenia treatment [2]. However, not all patients have the same access to all treatments. Generic medications are an important alternative for those who cannot afford branded agents, especially in developing nations [102]. However, it is also important to have fair, reasonable and evidence-based access to newer treatments without generic alternatives that have been shown to have an acceptable benefit-risk balance. The best people to make those decisions are the doctor and the patient, on the basis of the broadest possible information. When branded treatments are excluded, either by payers or court-assisted early genericization, there is no incentive for the development of new treatments. Extrapolated to the extreme, this could mean that new treatments will become a thing of the past if society is not prepared to pay the approximately US $\$ 1$ billion necessary to prove that a new drug meets acceptable safety and efficacy standards [3]. New drugs are not usually developed by the government with taxpayer dollars; they are developed by a private enterprise, which requires profit to exist [4]. This makes new drugs expensive to buy, but hopefully they offer reasonable value. Ultimately, their business will not exist if the customer is not happy, so there is a motivation to do the right thing for patients.

From time to time, drugs lose patent protection via court challenges. While in the short term this may be good news for patients and for the generic companies, in the long term it discourages investment in scientific innovation. Why would a company invest in a country where intellectual property protection can't be assured? In some countries where there is generic competition available for brand name drugs, the branded drug also continues to be used. Some might say this is a result of successful marketing, but a more relevant factor to physicians and patients is the potential for differences between a branded medication and its generic alternative.

$$
\begin{aligned}
& \text { "From a global perspective, } \\
& \text { it is no longer sufficient to simply } \\
& \text { study Caucasians when testing } \\
& \text { the bioavailability of a drug. Study } \\
& \text { samples should reasonably represent } \\
& \text { the full diversity of patients who } \\
& \text { might use the treatment." }
\end{aligned}
$$

Indeed, there have been reports of relapse or adverse events after a switch to a cheaper generic drug [5]. The reasons behind the differences in the use of branded or generic drugs in different countries should be considered. I suggest that, in part, it is politically more acceptable in North America to view branded pharmaceuticals with antipathy, while in many developing countries the cultural attitude is to follow the doctor's recommendation without question, whether it is to take bird's nest soup or a branded antipsychotic. Perhaps neither extreme is the wisest approach. Evidence-based prescribing is important even for generics drugs and the low level of evidence required to assume bioequivalence is problematic. Bioequivalence can be considered provided that as few as 20 patients have $\mathrm{C}_{\max }$, $\mathrm{T}_{\max }$ and area under the curve within a certain 
range [6]. Bioequivalence studies are designed to demonstrate similarities, not to disprove the possibility of differences [7]. This does not prevent occasional cases in which individual differences in pharmacodynamics result in different bioavailability, with consequent adverse events or efficacy loss.

From a global perspective, it is no longer sufficient to simply study Caucasians when testing the bioavailability of a drug. Study samples should reasonably represent the full diversity of patients who might use the treatment. Furthermore, this applies to randomized controlled trials (RCTs) and observational studies, as much as it should to bioequivalence studies.

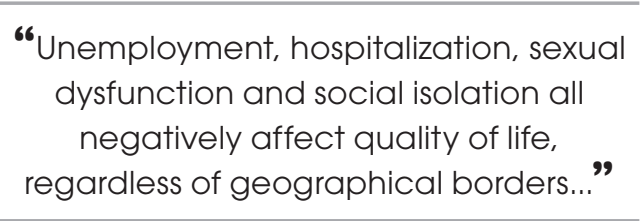

There has been considerable debate surrounding the issue of whether patients with schizophrenia in different parts of the world have different outcomes in response to treatment [8]. Most RCTs are carried out in the developed world, primarily North America and Europe [9]. For various reasons, RCTs are challenging to conduct in many developing countries [10], so it is difficult to determine whether trial results are generalizable to patients living in other parts of the world. RCTs are great for informing us about whether a drug can work under ideal circumstances, while observational studies help answer the question of whether a drug works under real-life conditions, which include global variations in dosing, polypharmacy practices, cultural attitudes towards doctors and medicines and availability or access to various treatments, both drug based and psychosocial.

Recently, an international study of over 17,000 individuals with schizophrenia reported striking similarities in symptoms, medication, employment and sexual problems, despite the fact that it covered a diverse range of patients and healthcare systems in 37 different countries [11]. The Worldwide-Schizophrenia Outpatient Health Outcomes (W-SOHO) study was a 3 -year observational study designed to assess costs and outcomes in outpatients using antipsychotics. Of the 37 countries in the study, 21 were emerging or developing economies, including those in East Asia, Latin America, North Africa and the Middle East, with the remainder coming from Europe.
The selection criteria were minimal, allowing a broad representation of 'real-world' patients. A total of 1563 clinicians took part in the study. Patients were assessed at baseline and at 3, 6, 12, 18,24 and 36 months. All clinicians taking part in the study were free to determine which treatment and medication their patients received and no drugs were provided by the study sponsor. Patients were drawn from a variety of economies, cultures and practice settings, yet were remarkably similar across the six regions in terms of demographic and clinical characteristics. However, there were also important differences noted in some areas and these differences have as much relevance to clinical practice as the similarities observed.

Despite some regional and country variations, the study demonstrated remarkably similar overall patterns in key health, medication and socioeconomic factors.

At baseline, the 17,384 patients, who were all starting or changing their antipsychotic medication, averaged 38 years of age and 57\% were male. The median duration of their illness was 7 years and $10 \%$ were antipsychotic naive. On average, only $19 \%$ were in paid employment, $69 \%$ were living in dependent housing and $62 \%$ had reported sexual problems in the previous month.

A total of $26 \%$ of the study participants were taking more than one antipsychotic and approximately $74 \%$ were taking other prescribed drugs at the same time. Similarly, in a Canadian observational study of schizophrenia, it was demonstrated that approximately $50 \%$ of patients were taking multiple antipsychotics [12]. Guidelines tend to frown upon polypharmacy in schizophrenia [13], but the persistence of this practice begs the question of whether it occurs for valid reasons.

\footnotetext{
"Observational studies, RCTs and

bioequivalence studies can be used

together to guide rational treatment choices in schizophrenia..."
}

The main reasons for changing medication were similar across all regions in the W-SOHO study, with two-thirds of the investigators citing lack of effectiveness, followed by intolerance, patient request and incomplete adherence to the medication. This supports similar findings in the Clinical Antipsychotic Trials of Intervention Effectiveness (CATIE) study [14], which, in contrast to prevailing opinions regarding the impact of adverse events, suggests that effective control of symptoms is the most important driver of new treatment choices. 
These patients were moderately ill overall, with an average Clinical Global Impression (CGI) severity score of 4.4 ( 1 being well and 7 being worst) and regional scores ranging from 3.9 to 4.7. Sexual dysfunction was common, averaging close to $60 \%$. With the exception of East Asia (33.5\%), regions reported similar levels of sexual dysfunction ranging from 57.9 to $67 \%$. Approximately $34 \%$ of patients had been admitted to an inpatient facility owing to their schizophrenia in the last 6 months. Individual regions showed very similar rates, ranging from 31.2 to $36.3 \%$, with the exception of Latin America (40.8\%). Approximately a quarter of the patients had attempted suicide at some time in their lives, with regional ranges from 21.7 to $30.1 \%$.

Paid employment status was consistently low, averaging $19 \%$ and ranging from 16.2 to $22.6 \%$. Just under a third of patients $(32.1 \%)$ were in a relationship, ranging from 25.1 to $38.6 \%$ in five of the six regions. However, people in East Asia were much more likely to be in a relationship (47.4\%).

These figures are sobering, and should give us cause to take into consideration the impact of this horrible disorder on the lives of the patient and their families more seriously. Unemployment, hospitalization, sexual dysfunction and social isolation all negatively affect quality of life [15,16], regardless of geographical borders, to say nothing of the distress and dysfunction caused by hallucinations and delusions [17]. It is beyond the scope of this article to compare the outcomes associated with various treatments in the W-SOHO study, but it should be suffice to say that in general, it confirms similar findings and is complementary to the findings of large RCTs.

It would be wise for people to be more open and less polarized in their views concerning the treatment of schizophrenia. Policy makers all over the world have an obligation to make the effort to understand and correctly interpret data

\section{Bibliography}

1. Saha S, Chant D, Welham J, McGrath J: A systematic review of the prevalence of schizophrenia. PLoS Med 2(5), E141 (2005).

2. Mueser KT, McGurk SR: Schizophrenia. Lancet 363(9426), 2063-2072 (2004).

3. DiMasi JA, Hansen RW, Grabowski HG: The price of innovation: new estimates of drug development costs. J. Health Econ. 22(2), 151-185 (2003).

that could help patients. Sometimes the data do actually contribute to smarter decisions, but in the future, we must do a better job of making prudent changes based on data we already have. Due consideration should be given to the needs of all nations for research and treatment related to schizophrenia. There is a need for cost-effective treatments for schizophrenia but there is also a need for the continued development of better treatments without the former eliminating the latter. Observational studies, RCTs and bioequivalence studies can be used together to guide rational treatment choices in schizophrenia, and can provide complementary information. For proper generalization and extrapolation of results, it is important that all of these studies are carried out with broadly diverse patient samples, ideally with multiple sites in different countries. Some countries will need help where the necessary research infrastructure is lacking. Policy makers across the globe must not only think about the short-term costs. They must become more transparent and consider the impact of their legal, regulatory and access decisions on the future health of the population. We must stop ignoring and misinterpreting valuable data. Treatment policies should be driven by data and not political concerns. There is merit to examining trends in clinical practice and healthcare policy, looking at consequences of past events to lead to wiser decisions.

\section{Financial \& competing interests disclosure \\ The opinions expressed in this editorial are the perspec- tives of the authors and do not necessarily reflect the opinions of Eli Lilly and Company. The authors have no other relevant affiliations or financial involvement with any organization or entity with a financial interest in or financial conflict with the subject matter or mate- rials discussed in the manuscript. This includes employ- ment, consultancies, honoraria, stock ownership or options, expert testimony, grants or patents received or pending, or royalties. \\ No writing assistance was utilized in the production of this manuscript.}

4. Pazderka B: The effect of pharmaceutical patent term length on research and development and drug expenditures in Canada. Healthc. Policy 2(3), 85-89 (2007).

5. Van Ameringen M, Mancini C, Patterson B, Bennett M: Symptom relapse following switch from Celexa to generic citalopram: an anxiety disorders case series.

J. Psychopharmacol. 21(5), 472-476 (2007).

6. Liu Y, Jia J, Liu G et al.: Pharmacokinetics and bioequivalence evaluation of two formulations of $10-\mathrm{mg}$ amlodipine besylate: an open-label, single-dose, randomized, two-way crossover study in healthy Chinese male volunteers. Clin. Ther. 31(4), 777-783 (2009).

7. Berry DA: Statistical Methodology in the Pharmaceutical Sciences. Marcel Dekker Inc., NY, USA, 70-71 (1990).

8. Cohen A, Patel V, Thara R, Gureje O: Questioning an axiom: better prognosis for schizophrenia in the developing world? Schizophr. Bull. 34, 229-244 (2008). 
9. Sheriff RJ, Adams CE, Tharyan P, Jayaram M, Duley L; PRACTIHC Mental Health Group: Randomised trials relevant to mental health conducted in low and middle-income countries: a survey. BMC Psychiatry 8, 69 (2008).

10. Smith R: Publishing research from developing countries. Stat. Med. 21(19), 2869-2877 (2002).

11. Karagianis J, Novick D, Haro JM et al:: Worldwide-Schizophrenia Outpatient Health Outcomes (W-SOHO): baseline characteristics of pan-regional observational data from more than 17,000 patients. Int. J. Clin. Pract. 63(11), 1578-1588 (2009).

12. Karagianis J, Williams R, Davis L et al.: Health outcomes of a Canadian community cohort: one year outcomes of outpatients with schizophrenia and related psychotic disorders who change to atypical antipsychotics. Current Med. Res. Opin. 225(9), 2121-2132 (2009).
13. Zink M, Englisch S, Meyer-Lindenberg A: Polypharmacy in schizophrenia. Curr. Opin. Psychiatry 23(2), 103-111 (2010).

14. Lieberman JA, Stroup TS, McEvoy JP et al.; Clinical Antipsychotic Trials of Intervention Effectiveness (CATIE) Investigators: Effectiveness of antipsychotic drugs in patients with chronic schizophrenia. N. Engl. J. Med. 353, 1209-1223 (2005).

15. Caron J, Mercier C, Diaz P, Martin A: Socio-demographic and clinical predictors of quality of life in patients with schizophrenia or schizo-affective disorder. Psychiatry Res. 137(3), 203-213 (2005).

16. Gasquet I, Tcherny-Lessenot S, Lépine JP, Falissard B: Patient satisfaction with psychotropic drugs: sensitivity to change and relationship to clinical status, quality-of-life, compliance and effectiveness of treatment. Results from a nation-wide 6-month prospective study. Eur. Psychiatry 21(8), 531-538 (2006).

17. Rocca P, Giugiario M, Montemagni C, Rigazzi C, Rocca G, Bogetto F: Quality of life and psychopathology during the course of schizophrenia. Compr. Psychiatry 50(6), 542-548 (2009).

\section{Websites}

101. WHO: The Global Burden of Disease: 2004 Update. www.who.int/healthinfo/global_burden_ disease/2004_report_update/en/index.html (Accessed February 2009).

102. Johnson T; Council on Foreign Relations: Generic Drugs: The Other Drug War. www.cfr.org/publication/18055/generic_ drugs.html

(Accessed 7 January 2010). 